\title{
ON GENERAL SURFACES DEFINED BY AN ALMOST LINEAR PFAFFIAN
}

\author{
LUCA CHIANTINI AND DANIELE FAENZI
}

\begin{abstract}
We calculate the integers $d$ such that a general surface $X_{d}$ in $\mathbb{P}^{3}$ of degree $d$ contains an arithmetically Gorenstein set of points with a linear syzygy matrix of size $2 \alpha+1$. This condition is equivalent to $X_{d}$ being defined by the pfaffian of a skew-symmetric matrix whose entries are linear except possibly a row and a column.

We prove that this takes place for all $d \geq \alpha+1$ if $\alpha \leq 10$. Conversely, for $\alpha \geq 11$, we show that the condition holds if and only if $d$ is contained in the interval $[\alpha+1,15]$.
\end{abstract}

\section{INTRODUCTION}

Let $X_{d}$ be an integral hypersurface of degree $d$ in $\mathbb{P}^{n}$, defined by a homoneneous polynomial $F$, with $n \geq 3$. Given an integer $r$, one can ask when is the polynomial $F^{r}$ defined by the determinant of a matrix $M$ whose entries are homogeneous polynomials of a given degree.

It is well understood that this condition is equivalent to the existence of a locally Cohen-Macaulay sheaf $\mathscr{E}$ of rank $r$, with a numerically determined resolution, on a scheme supported at $X_{d}$, which satisfies:

$$
\oplus_{m} \mathrm{H}^{i}\left(X_{d}, \mathscr{E}(m)\right)=0 \quad \text { for } i \neq 0, n-1,
$$

that is, the sheaf $\mathscr{E}$ is without intermediate cohomology. These sheaves are called arithmetically Cohen-Macaulay (aCM).

Assume now the hypersurface $X_{d}$ to be general, i.e. outside a subset of positive codimension of $\left|\mathscr{O}_{\mathbb{P}^{n}}(d)\right|$. Assume also the sheaf $\mathscr{E}$ to be not isomorphic to a direct sum of line bundles of the form $\mathscr{O}_{X_{d}}(t)$. For $d \geq 4$, Lefschetz's theorem implies $r \geq 2$. Hence the first case corresponds to rank 2 sheaves, and the matrix $M$ must be skew-symmetric. The determinant of $M$ is then the square of a polynomial called the pfaffian $\operatorname{Pf}(M)$ of $M$. In this case, arithmetically Gorenstein codimension 3 subschemes of $\mathbb{P}^{n}$ enter the picture (see below for the definition). Indeed, if $Z$ is one of these subschemes, according to [BE77] it has a skew-symmetric syzygy matrix $N_{Z}$. Now, if the hypersurface $X_{d}$ is the pfaffian of a matrix $M$, then $X_{d}$ contains a subscheme $Z \subset \mathbb{P}^{n}$ of codimension 3 and $N_{Z}$ is obtained by $M$ deleting one column and one row.

For $n=4$, the problem is settled in [Kle78, CM00, CM04, CM05, MKRR07a], MKRR07b]. The answer is that, for $d \geq 6$, no indecomposable rank 2 aCM sheaf is defined on a general hypersurface $X_{d} \subset \mathbb{P}^{n}$ of degree $d$, and so $X_{d}$ is not pfaffian. It follows that for higher $n$ the same statement holds too. Moreover, a classification is available for $d \leq 5$. For $n=5$, only the case $d=2$ survives, (the spinor bundles on a smooth quadric, see [Knö87, see also [tt88]). For $n \geq 6$, there is no such sheaf at all. 
It remains to take care of the case $n=3$. An effort in this direction is the classification of aCM bundles of rank 2 in case $d \leq 5$, see [Fae08a, CF08. The result is that for $d=3$ (resp. $d=4, d=5$ ) a general surface $X_{d} \subset \mathbb{P}^{3}$ of degree $d$ can be written as 3 different types (resp. 8 types, 14 types) of pfaffians.

A different approach is to fix, for each index $(i, j)$, the degree $d_{i, j}$ of the polynomial sitting on the $i$-th row and $j$-th column of the matrix $M$.

For instance we may set $d_{i, j}=1$ for all $i, j \geq 1$; we speak thus of a linear pfaffian. In [Bea00], it is proved (among many other things) that a general surface $X_{d}$ is a linear pfaffian iff $d \leq 15$. In [Fae08b], the same result is proved assuming $d_{i, j}=2$ for all $i, j \geq 2$.

In this paper, we study for which integers $d$, a general surface $X_{d}$ in $\mathbb{P}^{3}$ of degree $d$ is defined by the pfaffian of an almost linear skew-symmetric matrix $M$ of size $2 \alpha+2$, i.e. where at most one row and column have degree different from 1 . In this case we say that $X_{d}$ is an almost linear pfaffian of type $\alpha$. In terms of Hilbert schemes, we note that, once we fix an integer $\alpha \geq 1, X_{d}$ is an almost linear pfaffian of type $\alpha$ if and only if it contains a zero-dimensional subscheme $Z \subset \mathbb{P}^{3}$ whose syzygy matrix $N_{Z}$ has only linear entries and size $2 \alpha+1$.

Since every surface contains some points and any single point defines a zero-dimensional subscheme $Z \subset \mathbb{P}^{3}$ whose syzygy matrix $N_{Z}$ has only linear entries, then any surface is defined by the pfaffian of an almost linear skew-symmetric matrix (of type $\alpha=1$ ).

However, we want to discuss to which extent one can describe a general surface of degree $d$ as the pfaffian of an almost linear skew-symmetric matrix $M$, where $M$ has a preassigned type $\alpha$.

What we find is the following, somehow surprising result:

Theorem. A general surface $X_{d}$ is an almost linear pfaffian of type $\alpha$ for all $d \geq \alpha+1$ if $\alpha \leq 10$. Conversely, for $\alpha \geq 11$, the surface $X_{d}$ is an almost linear pfaffian if and only if $d$ is contained in the interval $[\alpha+1,15]$.

Notice that one can consider the previous result in the general setting of surfaces containing fixed types of zero-dimensional subschemes of $\mathbb{P}^{3}$. This amounts to fixing a component $T$ of the Hilbert scheme of subschemes of $\mathbb{P}^{3}$, and ask whether general surface $X_{d}$ in $\mathbb{P}^{3}$ of degree $d$ contains a subscheme $Z \in T$. If this happens for all $d \geq d_{0}$, we will say that $T$ is asymptotic (see definition 3.10).

The previous result thus proves that the Hilbert scheme of zero-dimension subschemes, with linear syzygy matrix of size $2 \alpha+1$, is asymptotic if and only if $\alpha \leq 10$.

It should be noted that, like in Schreyer's appendix of [Bea00], our proof that a general surface $X_{d}$ is defined by a pfaffian with fixed degree set $d_{i, j}$, when $d$ lies in a certain range, relies on a computation done with the package Macaulay2, see GS.

After recalling some basic notions, in the next section we introduce homogeneous subschemes and show how they are related to surfaces defined by an almost homogeneous pfaffian. In section 4 we prove our main results, while the appendix is devoted to the Macaulay2 scripts used in our computations. 
1.1. Notation and preliminary notions. We will work over an algebraically closed field $\mathbf{k}$. Given a scheme $Y$, embedded in $\mathbb{P}^{n}$ by the very ample line bundle $\mathscr{O}_{Y}(1)$, we denote by $R_{Y}$ the coordinate ring of $Y$, namely the quotient ring of $R=\mathbf{k}\left[x_{0}, \ldots, x_{n}\right]$ by the ideal $I_{Y}$ defining $Y$. The symbol $\mathscr{I}_{Z, Y}$ will stand for the ideal sheaf of a subscheme $Z$ of $Y$ (the letter $Y$ will be frequently dropped).

In this paper, the word resolution of a sheaf $\mathscr{F}$ over $\mathbb{P}^{n}$ will mean the sheafified minimal graded free resolution of the $R$-module $\oplus_{m} \mathrm{H}^{0}\left(\mathbb{P}^{n}, \mathscr{F}\right)$. Minimal here means that there are no nonzero constants in the differentials appearing in the resolution.

We will consider the Hilbert function $\mathrm{h}_{Y}(t)$ and its first and second difference function $\mathrm{g}_{Y}(t)$ and $\tau_{Y}(t)$, defined by:

$$
\begin{aligned}
\mathrm{h}_{Y}(t) & =\operatorname{dim}_{\mathbf{k}}\left(R_{Y}(t)\right), \\
\mathrm{g}_{Y}(t) & =\mathrm{h}_{Y}(t)-\mathrm{h}_{Y}(t-1) \\
\tau_{Y}(t) & =\mathrm{g}_{Y}(t)-\mathrm{g}_{Y}(t-1) .
\end{aligned}
$$

A subscheme $Y \subset \mathbb{P}^{n}$ is called arithmetically Gorenstein (aG) if $R_{Y}$ is a Gorenstein ring. Given a zero-dimensional subscheme $Z \subset \mathbb{P}^{n}$, we denote by $i_{Z}$ its index, namely the highest integer $i$ such that $\mathrm{h}_{Z}(i)<\operatorname{len}(Z)$ (where len $(Z)$ denotes the length of $Z$ ). Recall that the difference Hilbert function of an aG subscheme $Z$ satisfies $g_{Z}(t)=\mathrm{g}_{Z}\left(i_{Z}-t\right)$, for all integers $t$. Thus the function $g_{Z}$ is symmetric around the integer $j_{Z}=\left\lfloor\frac{i_{Z}}{2}\right\rfloor$. The integer $i_{Z}-1$ is called the socle degree of $Z$.

Recall that, by the structure theorem of [BE77, the ideal sheaf of an aG zero-dimensional subscheme $Z \subset \mathbb{P}^{3}$ has a resolution of the following form:

$$
0 \rightarrow \mathscr{O}_{\mathbb{P}^{3}}(-c) \rightarrow \bigoplus_{j=1}^{2 b+1} \mathscr{O}_{\mathbb{P}^{3}}\left(r_{j}-c\right) \stackrel{N_{Z}}{\longrightarrow} \bigoplus_{i=1}^{2 b+1} \mathscr{O}_{\mathbb{P}^{3}}\left(-r_{i}\right) \rightarrow \mathscr{I}_{Z, \mathbb{P}^{3}} \rightarrow 0
$$

and we have the equalities:

$$
i_{Z}=c-3, \quad c=\frac{\sum_{j=1}^{2 b+1} r_{j}}{b} .
$$

The map $N_{Z}$ is represented by a matrix with polynomial entries, which we still denote by $N_{Z}$. This matrix is skew-symmetric, and the ideal $I_{Z}$ is defined by all pfaffians of order $2 b$ of the matrix $N_{Z}$.

\section{Pfaffian Representations of SURfaces and aG SUbSchemes}

The existence of aG subschemes in a degree $d$ surface $X_{d} \subset \mathbb{P}^{3}$ is important inasmuch as it yields the existence of rank 2 bundles with no intermediate cohomology on $X_{d}$, i.e. aCM bundles (satisfying (1.1)). Equivalently, it determines a pfaffian representation of $X_{d}$, as pointed out by Beauville in [Bea00, see in particular Theorem B. The following theorem makes more precise the relationship between the existence of such a bundle over $X_{d}$ and the existence of aG subscheme of $X_{d}$ with specified resolution.

Theorem 2.1. Let $X_{d}=\mathbb{V}(F)$ be a smooth surface of degree $d$ in $\mathbb{P}^{3}$. Then the following conditions are equivalent: 
i) the surface $X_{d}$ contains an $a G$ subscheme $Z$, whose ideal sheaf has resolution

$$
0 \rightarrow \mathscr{P}_{2} \rightarrow \mathscr{P}_{1} \stackrel{N_{Z}}{\longrightarrow} \mathscr{P}_{0} \rightarrow \mathscr{I}_{Z, \mathbb{P}^{3}} \rightarrow 0,
$$

where there are non-negative integers $c, r_{1}, \ldots, r_{2 b+1}, b \geq 1$ with $c=$ $\left(\sum_{i=1}^{2 a+1} r_{i}\right) / b$ and:

$$
\mathscr{P}_{0}=\bigoplus_{i=1}^{2 b+1} \mathscr{O}_{\mathbb{P}^{3}}\left(-r_{i}\right), \quad \mathscr{P}_{1}=\bigoplus_{j=1}^{2 b+1} \mathscr{O}_{\mathbb{P}^{3}}\left(r_{j}-c\right), \quad \mathscr{P}_{2}=\mathscr{O}_{\mathbb{P}^{3}}(-c) .
$$

ii) the form $F$ is the pfaffian of a skew symmetric $(2 b+2) \times(2 b+2)$ matrix of forms $M=\left(m_{i j}\right)$, such that $\operatorname{deg}\left(m_{11}\right)=2 d-c, \operatorname{deg}\left(m_{1 j}\right)=$ $\operatorname{deg}\left(m_{j 1}\right)=d-r_{j-1}$ for $j>1$, and $\operatorname{deg}\left(m_{i j}\right)=c-r_{j-1}-r_{i-1}$ for $i, j>1$;

iii) on the surface $X_{d}$ there exists a rank 2 bundle $\mathscr{E}$ satisfying (1.1), with a resolution of the form:

$$
0 \rightarrow \mathscr{Q}_{1} \stackrel{M}{\longrightarrow} \mathscr{Q}_{0} \rightarrow \mathscr{E} \rightarrow 0
$$

with:

$$
\begin{aligned}
& \mathscr{Q}_{1}=\mathscr{O}_{\mathbb{P}^{3}}(c-2 d) \oplus \bigoplus_{j=1}^{2 b+1} \mathscr{O}_{\mathbb{P}^{3}}\left(r_{j}-d\right), \\
& \mathscr{Q}_{0}=\mathscr{O}_{\mathbb{P}^{3}} \oplus \bigoplus_{i=1}^{2 b+1} \mathscr{O}_{\mathbb{P}^{3}}\left(-r_{i}+c-d\right),
\end{aligned}
$$

and where the matrix representing the map $M$ is skew-symmetric. Moreover $Z$ is the zero locus of a section of $\mathscr{E}$, and the Chern classes of $\mathscr{E}$ are $c_{1}(\mathscr{E})=c-d, c_{2}(\mathscr{E})=\operatorname{len}(Z)$.

Proof. We prove the equivalence of (ii) and (iii). An aG subscheme $Z$ is a 0 -dimensional subscheme of $\mathbb{P}^{3}$ which is locally complete intersection, and the resolution of the ideal sheaf $\mathscr{I}_{Z}$ is of type (2.1).

One sees easily that the resolution (2.1) determines an element $\xi$ of the group:

$$
\operatorname{Ext}_{\mathbb{P}^{3}}^{2}\left(\mathscr{I}_{Z}, \mathscr{O}_{\mathbb{P}^{3}}(-c)\right) \cong \mathrm{H}^{1}\left(\mathbb{P}^{3}, \mathscr{I}_{Z}(c-4)\right)^{*},
$$

where the above isomorphism is given by by Serre duality. Notice that $\xi$ comes from the map $\mathscr{O}_{\mathbb{P}^{3}} \rightarrow \mathscr{E} x t_{\mathbb{P}^{3}}^{2}\left(\mathscr{I}_{Z}, \mathscr{O}_{\mathbb{P}^{3}}(-c)\right)$ which is locally surjective, for $Z$ is locally complete intersection.

The exact sequence:

$$
0 \rightarrow \mathscr{O}_{\mathbb{P}^{3}}(-d) \rightarrow \mathscr{I}_{Z} \rightarrow \mathscr{I}_{Z, X_{d}} \rightarrow 0,
$$

(where $\left.\mathscr{O}_{\mathbb{P}^{3}}(-d)=\mathscr{I}_{X_{d}}\right)$ gives the isomorphism $\mathrm{H}^{1}\left(\mathbb{P}^{3}, \mathscr{I}_{Z}(c-4)\right) \cong$ $\mathrm{H}^{1}\left(\mathbb{P}^{3}, \mathscr{I}_{Z, X_{d}}(c-4)\right)$. By Serre duality again, this time on $X_{d}$, we get that $\xi$ determines an element $\xi_{X_{d}}$ of $\operatorname{Ext}_{X_{d}}^{1}\left(\mathscr{I}_{Z, X_{d}}, \mathscr{O}_{X_{d}}(d-c)\right)$ which is a local generator of the sheaf $\mathscr{E} x t_{X_{d}}^{1}\left(\mathscr{I}_{Z, X_{d}}, \mathscr{O}_{X_{d}}(d-c)\right)$. Thus the element $\xi_{X_{d}}$ yields an exact sequence:

$$
0 \rightarrow \mathscr{O}_{X_{d}} \rightarrow \mathscr{E} \rightarrow \mathscr{I}_{Z, X_{d}}(c-d) \rightarrow 0
$$

where $\mathscr{E}$ is locally free on $X_{d}$, since we are assuming that $X_{d}$ is smooth. 
Now, since $\xi$ and $\xi_{S}$ are corresponding elements, these two sequences patch together giving an exact diagram:

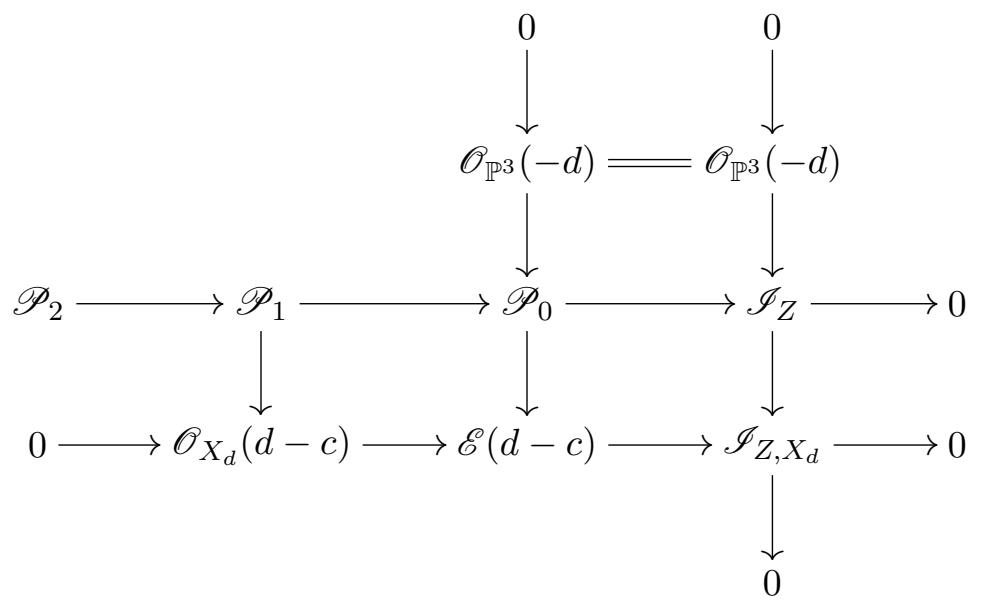

The mapping cone now determines a resolution:

$$
0 \rightarrow \mathscr{O}_{\mathbb{P}^{3}}(-d) \oplus \mathscr{P}_{1} \rightarrow \mathscr{O}(d-c) \oplus \mathscr{P}_{0} \rightarrow \mathscr{E}(d-c) \rightarrow 0,
$$

and twisting by $\mathscr{O}_{\mathbb{P}^{3}}(c-d)$ we get $(2.2)$. We have thus proved (ii) $\Rightarrow$ (iii).

Conversely, assume $\mathscr{E}$ is a rank 2 bundle on $S$, with a resolution as in (2.2) and recall the exact sequence:

$$
0 \rightarrow \mathscr{O}_{\mathbb{P}^{3}}(-d) \rightarrow \mathscr{O}_{\mathbb{P}^{3}} \rightarrow \mathscr{O}_{X_{d}} \rightarrow 0,
$$

We get a commutative diagram:

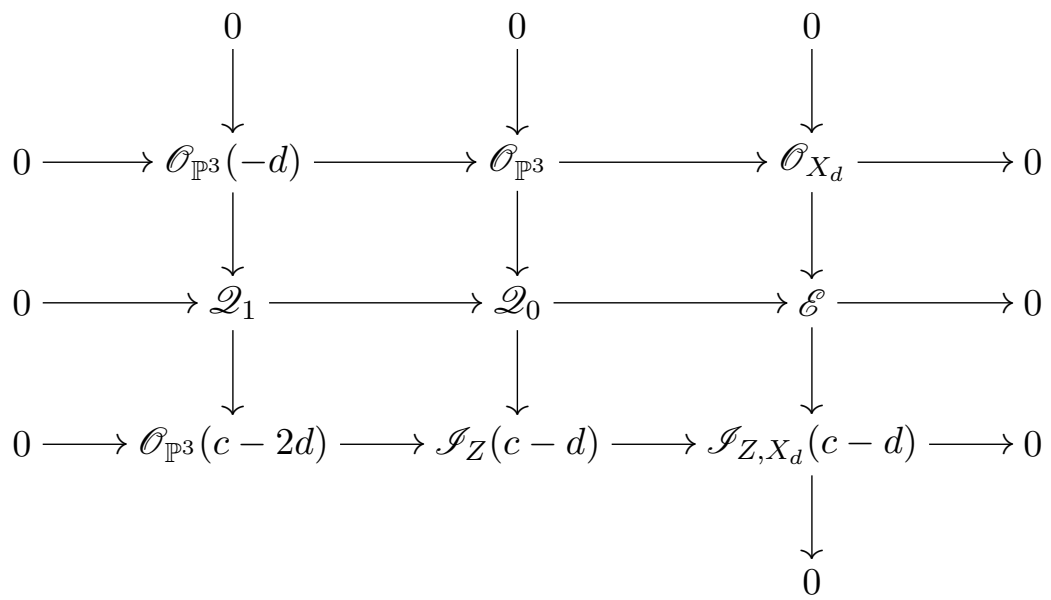

This diagram, by the mapping cone again, after twisting by $\mathscr{O}_{\mathbb{P}^{3}}(c-d)$ determines a resolution of the form:

$$
0 \rightarrow \mathscr{O}_{\mathbb{P}^{3}}(-c) \rightarrow \mathscr{O}_{\mathbb{P}^{3}}(d-c) \oplus \mathscr{Q}_{1}(d-c) \stackrel{N_{Z}^{\prime}}{\longrightarrow} \mathscr{O}_{\mathbb{P}^{3}}(-d) \oplus \mathscr{Q}_{0}(d-c) \rightarrow \mathscr{I}_{Z} \rightarrow 0 .
$$

We may decompose the matrix $N_{Z}^{\prime}$ into blocks according to the splitting of the bundle $\mathscr{Q}_{0}(d-c)$ (respectively, of $\mathscr{Q}_{1}(d-c)$ ) into the three summands 
$\mathscr{O}_{\mathbb{P}^{3}}(-d), \mathscr{O}_{\mathbb{P}^{3}}(d-c)$ and $\bigoplus \mathscr{O}_{\mathbb{P}^{3}}\left(-r_{j}\right)$ (respectively, according to the duality $\left.\mathscr{Q}_{1} \cong \mathscr{Q}_{0}^{*}(c-2 d)\right)$.

We get a decomposition of the form:

$$
N_{Z}^{\prime}=\left(\begin{array}{ccc}
0 & \lambda & 0 \\
-\lambda & 0 & n \\
0 & -n^{\top} & N_{Z}
\end{array}\right),
$$

where the matrix $N_{Z}$ has the expression given in the exact sequence (2.1), and the block $n$ corresponds to the mapping $\bigoplus \mathscr{O}_{\mathbb{P} 3}\left(r_{j}-c\right) \rightarrow \mathscr{O}_{\mathbb{P} 3}(d-c)$. Indeed, the mapping $\mathscr{O}_{\mathbb{P}^{3}}(d-c) \hookrightarrow \mathscr{O}_{\mathbb{P}^{3}}(-d) \oplus \mathscr{Q}_{0}(d-c)$ is given by the embedding $\mathscr{O}_{\mathbb{P}^{3}} \hookrightarrow \mathscr{Q}_{0}$, and we can choose a basis of $\mathscr{Q}_{0}$ where this takes the form $(\lambda, 0, \ldots, 0)$. In particular, the scalar $\lambda$ corresponding to the mapping $\mathscr{O}_{\mathbb{P}^{3}}(d-c) \rightarrow \mathscr{O}_{\mathbb{P}^{3}}(d-c)$ is nonzero.

In view of the decomposition (2.4), and since $Z$ is contained in $X_{d}=$ $\mathbb{V}(\operatorname{Pf}(M))$, the ideal generated by the pfaffians of order $2 a+2$ of $N_{Z}^{\prime}$ coincides with the ideal generated by the pfaffians of order $2 a$ of $N_{Z}$. We have thus obtained (2.1), and we note that $N_{Z}$ is minimal as soon as $M_{Z}$ is so. Observe that the subscheme $Z$ is locally complete intersection, since it is the 0-locus of a section of a rank 2 bundle on $S$. This implies that $Z$ is aG.

Finally, (iii) implies (ii), for the map $M$ can be represented as a skewsymmetric matrix whose entries have the required degree. The converse is proved in Bea00, Corollary 2.4]

\section{Homogeneous Subschemes And ALmost homogeneous PFAFFian SURFACES}

3.1. Homogeneous subschemes. A particularly interesting class of aG subschemes of $\mathbb{P}^{3}$ is that of homogeneous subschemes. Namely, it consists of those subschemes $Z \subset \mathbb{P}^{3}$ whose syzygy matrix $N_{Z}$ in the resolution 1.5 has entries which are homogeneous polynomials of the same degree.

Definition 3.1. Given two integers $\alpha, \beta \geq 1$, we say that an aG subscheme $Z \subset \mathbb{P}^{3}$ is homogeneous of degree $\beta$ and order $\alpha$ if the sheafified minimal graded free resolution of $\mathscr{I}_{Z}$ takes the form:

(3.1) $0 \rightarrow \mathscr{O}_{\mathbb{P}^{3}}(-(2 \alpha+1) \beta) \rightarrow \mathscr{O}_{\mathbb{P}^{3}}(-(\alpha+1) \beta)^{2 \alpha+1} \stackrel{N_{Z}}{\longrightarrow} \mathscr{O}_{\mathbb{P}^{3}}(-\alpha \beta)^{2 \alpha+1} \rightarrow \mathscr{I}_{Z, \mathbb{P}^{3}} \rightarrow 0$.

With the notation of $(1.5)$, this amounts to require that the matrix $N_{Z}$ has size $(2 \alpha+1) \times(2 \alpha+1)$ and all of its entries have the same degree $\beta$. If moreover $\beta=1$, we will say that $Z$ is linearly homogeneous of order $\alpha$.

It is clear, from the definition, that the easiest example of homogeneous aG scheme is given by a single point (here $\alpha=\beta=1$ ).

It follows from the definition that the length and the index of a homogeneous aG subscheme of degree $\beta$ and order $\alpha$ are:

$$
\begin{aligned}
& \operatorname{len}(Z)=\ell_{\alpha}^{\beta}=\frac{\alpha \beta^{3}\left(2 \alpha^{2}+3 \alpha+1\right)}{6}, \\
& i_{Z}=i_{\alpha}^{\beta}=(2 \alpha+1) \beta-3 .
\end{aligned}
$$

It is easy to calculate the first and second difference functions of a homogeneous subscheme. 
Lemma 3.2. Let $Z \subset \mathbb{P}^{3}$ be homogeneous of degree $\beta$ and order $\alpha$. When $\beta \geq 2$, then the second difference function of $Z$ takes the form:

$$
\tau_{Z}=\tau_{\alpha}^{\beta}(t)= \begin{cases}t+1, & \text { for } 0 \leq t \leq \alpha \beta-1 \\ \max \{\alpha \beta-2 \alpha(t-\alpha \beta+1), 0\}, & \text { for } \alpha \beta \leq t \leq \alpha \beta+\frac{\beta-1}{2} .\end{cases}
$$

The first difference function of a linearly homogeneous subscheme $Z$ takes the form:

$$
\mathrm{g}_{Z}=\mathrm{g}_{\alpha}^{1}(t)=\left\{\begin{array}{cl}
\left(\begin{array}{c}
t+2 \\
2
\end{array}\right) & \text { for } 0 \leq t \leq \alpha-1 \\
\left(\begin{array}{c}
\alpha-t \\
2
\end{array}\right) & \text { for } \alpha \leq t \leq 2 \alpha-2 .
\end{array}\right.
$$

Proof. Note that value of $g_{Z}(t)$ agrees with that of $g_{\mathbb{P} 3}(t)$, for all $t \leq \alpha \beta-$ 1 , indeed for any such $t$ we have $\mathrm{H}^{0}\left(\mathbb{P}^{3}, \mathscr{I}_{Z}(t)\right)=0$. This is enough to determine $\mathrm{g}_{Z}$ in case $\beta=1,2$ and in particular (3.5) follows.

For (3.4), one has to consider that the resolution of the ideal of $Z$ implies moreover $\mathrm{h}_{Z}(t)=\mathrm{h}_{\mathbb{P}^{3}}(t)-(2 \alpha+1) \mathrm{h}_{\mathbb{P}^{3}}(t-\alpha \beta)$ from $t=\alpha \beta$ to $t=\alpha \beta+\frac{\beta-1}{2}$, and this is again enough to determine the second difference of the Hilbert function anywhere.

Now we consider the Hilbert scheme containing homogeneous subschemes. Its dimension is well known, let us recall it in the next lemma.

Lemma 3.3. Given two integers $\alpha, \beta \geq 1$, there is a unique irreducible component $\mathscr{G}_{\beta}^{\alpha}$ of the Hilbert scheme of subschemes of $\mathbb{P}^{3}$ which contains all homogeneous subschemes of degree $\beta$ and order $\alpha$. We have:

$$
\operatorname{dim}\left(\mathscr{G}_{\beta}^{\alpha}\right)=\frac{1}{6}(2 \alpha+1)\left(\alpha \beta^{3}+6 \alpha \beta^{2}+11 \alpha \beta-6 \alpha-6\right) .
$$

A subscheme corresponding to a general point in $\mathscr{G}_{\beta}^{\alpha}$ is homogeneous of degree $\beta$ and order $\alpha$.

Proof. The set of zero-dimensional subschemes of $\mathbb{P}^{3}$ which have the same Hilbert function is an irreducible variety, see Die96. Since all aG homogeneous subschemes, of degree $\beta$ and order $\alpha$, have the same Hilbert function, they are contained in a unique irreducible variety, over which the Hilbert function is constant. We define $\mathscr{G}_{\beta}^{\alpha}$ to be this variety.

Several formulas are then available to compute the dimension of $\mathscr{G}_{\beta}^{\alpha}$, see for instance [Kle98]. In order to use these formulas, one first has to compute the resolution of a general element in $\mathscr{G}_{\beta}^{\alpha}$. This can be done as in [Die96] (but see also the book [K99, section 5). For given $\alpha, \beta$, using the formulas of lemma 3.2 and the formulas of [IK99], one obtains that the ideal sheaf of a general element in $\mathscr{G}_{\beta}^{\alpha}$ has exactly the resolution described in 3.1. Hence it is homogeneous of order $\alpha$ and degree $\beta$. Then we use the formulas of [Kle98, and the claim on the dimension of $\mathscr{G}_{\beta}^{\alpha}$ follows.

Remark 3.4. Another quick way to obtain that the dimension of $\mathscr{G}_{\beta}^{\alpha}$ is the one given in (3.6), is the following. Recall that any subscheme $Z \subset \mathbb{P}^{3}$ of the required form is determined by a skew-symmetric matrix $N_{Z}$ of order $2 \alpha+1$. The entries of the matrix $N_{Z}$ are homogeneous forms on $\mathbb{P}^{3}$ of degree $\beta$. The set of such matrices is in bijection with $\wedge^{2} W \otimes \operatorname{Sym}^{\beta} V$, where $V$ and $W$ are vector spaces respectively of dimension $4,2 \alpha+1$. Note that $\mathrm{GL}(W)$ acts on this set, and the stabilizer of a general point is trivial. The dimension of this 
space thus equals (3.6). Clearly, all matrices lying in the same $\mathrm{GL}(W)$-orbit correspond to the same subscheme, hence an open dense subset of $\mathscr{G}_{\beta}^{\alpha}(t)$ is in bijection with $\wedge^{2} W \otimes \operatorname{Sym}^{\beta} V / \mathrm{GL}(W)$. Thus, the dimension of this set equals $\operatorname{dim}\left(\mathscr{G}_{\beta}^{\alpha}\right)$.

3.2. Almost homogeneous pfaffian surfaces. Assume that a degree $d$ surface $X_{d} \subset \mathbb{P}^{3}$ is defined as the pfaffian of a matrix $M$. Then we know that $X_{d}$ contains some aG subscheme $Z \subset \mathbb{P}^{3}$.

The case that $Z$ is homogeneous corresponds to the case that $M$ is a matrix of homogeneous polynomials of the same degree, except at most one row and one column.

Definition 3.5. Let $\alpha, \beta \geq 1$ and $\gamma$ be integers. Consider a skew-symmetric injective map:

$$
\mathscr{O}_{\mathbb{P}^{3}}(-\beta-2 \gamma) \oplus \mathscr{O}_{\mathbb{P}^{3}}(-\beta-\gamma)^{2 \alpha+1} \stackrel{M}{\longrightarrow} \mathscr{O}_{\mathbb{P}^{3}} \oplus \mathscr{O}_{\mathbb{P}^{3}}(-\gamma)^{2 \alpha+1} .
$$

We denote again by $M$ the matrix representing this map, and we assume that $M$ is minimal, i.e. that every constant entry of $M$ is zero. We denote by $\operatorname{Pf}_{k}(M)$ the ideal generated by pfaffians of $M$ of order $k$, while $\operatorname{Pf}(M)$ denotes the pfaffian of maximal order $2 \alpha+2$ of $M$. A form $F$ which equals $\operatorname{Pf}(M)$, for some matrix $M$ of this form, is said to be an almost homogeneous pfaffian of type $(\alpha, \beta, \gamma)$. The matrix $M$ itself is called an almost homogeneous map of type $(\alpha, \beta, \gamma)$. We have:

$$
\operatorname{deg}(\operatorname{Pf}(M))=\alpha \beta+\beta+\gamma .
$$

Note that, since $M$ is minimal, if $\operatorname{Pf}(M)$ is not zero we must have:

$$
\beta+\gamma \geq 1, \quad \text { so: } \operatorname{deg}(\operatorname{Pf}(M)) \geq \alpha \beta+1 .
$$

If $\beta=1$, we say that $\operatorname{Pf}(M)$ is an almost linear pfaffian of type $(\alpha, \gamma)$, or simply of order $\alpha$. When $\gamma=0$, and consequently $\operatorname{deg}(\operatorname{Pf}(M))=\alpha \beta+\beta$, we will say that $M$ is a homogeneous map of type $(\alpha, \beta)$ and $\operatorname{Pf}(M)$ is called a homogeneous pfaffian of type $(\alpha, \beta)$. If furthermore $\beta=1$, we say that $\operatorname{Pf}(M)$ is a linear pfaffian of order $\alpha$.

Remark 3.6. With the notation of Theorem 2.1, the following conditions are equivalent:

i) the form $F$ is the pfaffian of a skew symmetric homogeneous map $f$ of type $(\alpha, \beta, \gamma)$, and $\operatorname{Pf}_{2 \alpha-2}(f)$ is not contained in $(F)$.

ii) on the surface $X_{d}$ it is defined a rank 2 aCM bundle $\mathscr{E}$ with $c_{1}(\mathscr{E})=$ $\alpha \beta-\gamma$, admitting a skew-symmetric resolution of the form:

$0 \rightarrow \mathscr{O}_{\mathbb{P}^{3}}(-\beta-2 \gamma) \oplus \mathscr{O}_{\mathbb{P}^{3}}(-\beta-\gamma)^{2 \alpha+1} \rightarrow \mathscr{O}_{\mathbb{P}^{3}} \oplus \mathscr{O}_{\mathbb{P}^{3}}(-\gamma)^{2 \alpha+1} \rightarrow \mathscr{E} \rightarrow 0$.

iii) the surface $X_{d}$ has degree $\alpha \beta+\beta+\gamma$ and contains a homogeneous aG subscheme $Z$ of type $(\alpha, \beta)$;

So we will consider from now on almost homogeneous pfaffian surfaces. Since every surface contains some points, it is clear that any surface is almost homogeneous.

However, one point determines a $4 \times 4$ almost linear pfaffian representation of the surface in which the non-linear row (and column) has degrees $(2 d-$ $3, d-1, d-1, d-1)$. 
We will show in the next sections that, except for few cases, one cannot find almost homogeneous pfaffian representations of general surfaces of high degree, where the size of the matrix is arbitrary.

3.3. Asymptotic subschemes. Given a family of subschemes of $\mathbb{P}^{3}$, it is reasonable to ask whether a general element of the linear system $\left|\mathscr{O}_{\mathbb{P}^{3}}(d)\right|$ contains an element of the family. In particular, given integers $(\alpha, \beta, \gamma)$, one can ask whether a general surface $X_{d} \subset \mathbb{P}^{3}$ of degree $d$ is an almost homogeneous pfaffian of type $(\alpha, \beta, \gamma)$. Accordingly, one can ask whether this is the case for big enough $d$. For instance, we have the following results.

Remark 3.7. Every surface $X_{d}$ of degree $d$ is defined by an almost linear pfaffian of type $(1, d-2)$. Indeed $X_{d}$ contains one point $Z$, which is a homogeneous aG subscheme with resolution of the form:

$$
0 \rightarrow \mathscr{O}_{\mathbb{P}^{3}}(-3) \rightarrow \mathscr{O}_{\mathbb{P}^{3}}(-2)^{3} \stackrel{N_{Z}}{\longrightarrow} \mathscr{O}_{\mathbb{P}^{3}}(-1)^{3} \rightarrow \mathscr{I}_{Z} \rightarrow 0 .
$$

On the other hand, it is much harder to get surfaces which are defined by homogeneous pfaffians. This is illustrated by the results of BeauvilleSchreyer and the second author:

Theorem 3.8. Let $X_{d} \subset \mathbb{P}^{3}$ be a general surface of degree $d$.

i) Let $d=\alpha+1$. Then $X_{d}$ is defined by a linear pfaffian of order $\alpha$ if and only if $\alpha \leq 14$, i.e. iff $d \leq 15$.

ii) Let $d=2 \alpha+2$. Then $X_{d}$ is defined by a homogeneous pfaffian of type $(\alpha, 2)$ if and only if $\alpha \leq 6$ i.e. $d \leq 14$.

Proof. For the first assertion, see [Bea00], in particular see Schreyer's appendix for the "if" implication. The second statement is the content of [Fae08b].

We recall here the definition given in the introduction.

Definition 3.9. A subscheme $T$ of the Hilbert scheme of $\mathbb{P}^{3}$ is called degreeasymptotic (or simply asymptotic) if there exists an integer $d_{0}$ such that for all $d \geq d_{0}$, a general surface $X_{d}$ in $\mathbb{P}^{3}$ of degree $d$ contains a subscheme $Z \in T$.

We will be mainly interested in the case where $T$ parametrizes aG subschemes. In particular, we will consider the case in which $T$ is the component of the Hilbert scheme parametrizing 0-dimensional sets $Z \subset \mathbb{P}^{3}$ with fixed Hilbert function. This depends only on the Hilbert function itself, or equivalently on the first difference function. We give thus the following definition.

Definition 3.10. A function $g: \mathbb{N} \rightarrow \mathbb{N}$ is called degree-asymptotic (or just asymptotic) if the subscheme $T$ of the Hilbert scheme parametrizing 0 -dimensional schemes $Z$ having $\mathrm{g}_{Z}=g$, is degree-asymptotic.

In other words, $g: \mathbb{N} \rightarrow \mathbb{N}$ is degree-asymptotic if there exists an integer $d_{0}$ such that for all $d \geq d_{0}$, a general surface $X_{d}$ in $\mathbb{P}^{3}$ of degree $d$ contains a subscheme $Z \subset \mathbb{P}^{3}$ with $g_{Z}=g$. A subscheme $Z \subset \mathbb{P}^{3}$ is degree-asymptotic if and only if its first difference function $g_{Z}$ is degree-asymptotic.

The following proposition follows a heuristic argument of M. Noether. Despite its rather simple proof, it often turns out to be useful. 
Proposition 3.11. Let $\mathrm{h}_{Z}$ and $\mathrm{g}_{Z}$ be respectively the Hilbert function and the first difference function of an aG subscheme $Z \subset \mathbb{P}^{3}$, and let $G$ be the component of the Hilbert scheme of subschemes of $\mathbb{P}^{3}$ which contains $[Z]$.

Assume that a general surface $X_{d} \subset \mathbb{P}^{3}$ of degree d contains a subscheme lying in $G$. Then we have:

$$
\mathrm{h}_{Z}(d) \leq \operatorname{dim}(G) .
$$

In particular, the function $g$ is degree-asymptotic only if:

$$
\operatorname{len}(Z)=\sum_{i=0}^{\infty} \mathrm{g}_{Z}(i) \leq \operatorname{dim}(G) .
$$

Proof. Consider the incidence variety:

$$
G(d)=\left\{\left(X_{d},[Z]\right) \mid Z \subset X_{d} \subset \mathbb{P}^{3}\right\} \subset\left|\mathscr{O}_{\mathbb{P}^{3}}(d)\right| \times G,
$$

and the projections $q_{d}: G(d) \rightarrow G$ and $p_{d}: G(d) \rightarrow\left|\mathscr{O}_{\mathbb{P}^{3}}(d)\right|$.

Looking at the $q_{d}$-fibre over a homogeneous subscheme $[Z]$, we note that it consists of the projectivized space $\mathbb{P}\left(\mathrm{H}^{0}\left(\mathbb{P}^{3}, \mathscr{I}_{Z}(d)\right)\right)$, hence its dimension equals $\mathrm{h}^{0}\left(\mathbb{P}^{3}, \mathscr{O}_{\mathbb{P}^{3}}(d)\right)-\mathrm{h}_{Z}(d)-1$. Assuming now the map $p_{d}$ to be dominant, we should have:

$$
\begin{aligned}
\mathrm{h}^{0}\left(\mathbb{P}^{3}, \mathscr{O}_{\mathbb{P}^{3}}(d)\right)-1= & \operatorname{dim}\left|\mathscr{O}_{\mathbb{P}^{3}}(d)\right| \leq \operatorname{dim}(G(d)) \leq \\
& \leq \operatorname{dim}(G)+\operatorname{dim}\left(q_{d}^{-1}([Z])\right),
\end{aligned}
$$

and this implies at once $(3.9)$. To prove (3.10), just note that $\mathrm{h}_{Z}(m)=$ $\sum_{i=0}^{m} \mathrm{~g}_{Z}(i)=\operatorname{len}(Z)$ for $m \gg 0$.

We will see below that the previous implication in general cannot be reversed, see Remark 4.6 .

\section{General surfaces as almost homogeneous pfaffians}

This section contains our main results. Namely, we study the problem of whether a general surface of degree $d$ is an (almost) homogeneous pfaffian of fixed type, with special attention to the linear almost homogeneous case.

4.1. Almost homogeneous pfaffian surfaces. Let us start with a result on homogeneous pfaffians of degree higher than one.

Theorem 4.1. Let $\beta \geq 2$ be an integer. For $\alpha \geq 8$, a general surface of degree $d=\alpha \beta+\beta+\gamma$ is not defined by an almost homogeneous pfaffian of type $(\alpha, \beta, \gamma)$, for any $\gamma \in \mathbb{Z}$.

Proof. The proof is essentially an application of Proposition 3.11. Namely, a homogeneous aG subscheme $Z$ of type $(\alpha, \beta)$ has a resolution of the form (3.1). Recall that, by the minimality of the matrix $M$ appearing in our definition of an almost homogeneous pfaffian (Definition 3.5), we have $\beta+$ $\gamma \geq 1$. Since the degree $d$ of the surface satisfies $d=\alpha \beta+\beta+\gamma$, we have:

$$
\mathrm{h}_{Z}(d)=\mathrm{h}_{Z}(\alpha \beta+\beta+\gamma) \geq \mathrm{h}_{Z}(\alpha \beta+1) .
$$

In view of Proposition 3.11 , it suffices to prove that the difference:

$$
\mathrm{h}_{Z}(\alpha \beta+\beta+\gamma)-\operatorname{dim}\left(\mathscr{G}_{\beta}^{\alpha}\right)
$$


is strictly positive, in the given range for $\alpha, \beta$ and for any $\gamma$. So by (4.1) it suffices to prove that the function:

$$
\varphi(\alpha, \beta)=\mathrm{h}_{Z}(\alpha \beta+1)-\operatorname{dim}\left(\mathscr{G}_{\beta}^{\alpha}\right)
$$

is strictly positive, in the given range for $\alpha, \beta$.

Now we use the argument of Lemma 3.2 , to prove:

$$
\mathrm{h}_{Z}(\alpha \beta+1)=\mathrm{h}_{\mathbb{P}^{3}}(\alpha \beta+1)-4(2 \alpha+1)=\left(\begin{array}{c}
\alpha \beta+4 \\
3
\end{array}\right)-4(2 \alpha+1) .
$$

Indeed, twisting (3.1) by $\mathscr{O}_{\mathbb{P} 3}(\alpha \beta+1)$ and taking global sections, we obtain $4(2 \alpha+1)$ independent elements in $\mathrm{H}^{0}\left(\mathbb{P}^{3}, \mathscr{I}_{Z, \mathbb{P}^{3}}(\alpha \beta+1)\right.$ ), and (since $\beta \geq 2$ ) no syzygies. Therefore, using (3.6) we can calculate the function $\varphi(\alpha, \beta)$ and find out:

$\varphi(\alpha, \beta)=\frac{1}{6}\left(\alpha^{3} \beta^{3}-2 \alpha^{2} \beta^{3}-3 \alpha^{2} \beta^{2}-\alpha \beta^{3}-22 \alpha^{2} \beta-6 \alpha \beta^{2}+12 \alpha^{2}+15 \alpha \beta-30 \alpha+6\right)$.

Deriving twice $\varphi$ with respect to $\alpha$ one finds:

$$
\frac{1}{3}\left(3 \alpha \beta^{3}-2 \beta^{3}-3 \beta^{2}-22 \beta+12\right),
$$

so $\partial \varphi / \partial \alpha$ is increasing in $\alpha$ for $\alpha \geq \alpha_{0}$ with:

$$
\alpha_{0}=\frac{2}{3}+\frac{1}{\beta}+\frac{22}{3 \beta^{2}}-\frac{4}{\beta^{3}} .
$$

Note that, since $\beta \geq 2$, we have $\alpha_{0} \leq 3$ so $\partial \varphi / \partial \alpha$ is increasing in $\alpha$ in our range. Now we compute $\varphi(8, \beta)$ and $\partial \varphi / \partial \alpha(8, \beta)$. Computing the second derivative in $\beta$ of these functions one sees that they are both convex for $\beta \geq 2$. Likewise, one checks that they are both increasing at $\beta=2$ and finally that they are both positive again at $\beta=2$. Thus $\varphi(\alpha, \beta)$ is positive for $\alpha \geq 8$ and $\beta \geq 2$, and the theorem is proved.

4.2. Almost linear pfaffian surfaces: the non-asymptotic region. In the remaining part of this section, we describe the linear almost homogeneous case, i.e. the case $\beta=1$. Namely, we determine all the values of $\alpha, \gamma$ for which a general surface of degree $d=\alpha+\gamma+1$ is defined by an almost homogeneous pfaffian of type $(\alpha, 1, \gamma)$. We start with the following, easy result.

Lemma 4.2. A surface of degree $d$ is not defined by an almost linear pfaffian of order $\alpha$, for $\alpha \geq d$.

Proof. Assume that a surface $X \subset \mathbb{P}^{3}$ of degree $d$ is defined by an almost linear pfaffian of order $\alpha$. Then, according to Definition 3.5, there must be a minimal matrix $M$ of the form 3.7 such that $X$ is defined by $\operatorname{Pf}(M)$. Since $\operatorname{Pf}(M) \neq 0$, minimality of $M$ easily implies that $\beta+\gamma \geq 1$, which means $\gamma \geq 0$ since $\beta=1$. Recall that the degree $d$ of $X$ satisfies $d=\alpha \beta+\beta+\gamma=$ $\alpha+\gamma+1$. We have thus proved that $d \geq \alpha+1$.

The next proposition establishes our main result for $\alpha \geq 16$.

Proposition 4.3. Fix an integer $\alpha \geq 16$. Then, for any integer $d$, a general surface of degree $d$ is not defined by an almost linear pfaffian of order $\alpha$. It follows that, when $\alpha \geq 16$, homogeneous subschemes $Z$ of type $(\alpha, 1)$ are not asymptotic. 
Proof. In view of the previous Lemma, we must simply exclude the case $d \geq \alpha+1$. Looking back at the proof of Proposition 3.11, we consider the following function of $(d, \alpha)$

$$
\mathrm{h}_{Z}(d)-\operatorname{dim}\left(\mathscr{G}_{1}^{\alpha}\right),
$$

and we prove that it is strictly positive in our range. Note that $\operatorname{dim}\left(\mathscr{G}_{1}^{\alpha}\right)=$ $4 \alpha^{2}-1$ by (3.6). It is easy to see that the function of $\alpha$

$$
\mathrm{h}_{Z}(\alpha+1)-\operatorname{dim}\left(\mathscr{G}_{1}^{\alpha}\right)
$$

is positive for $\alpha \geq 16$. Then a general surface of degree $\alpha+1$ contains no homogeneous subschemes of type $(\alpha, 1)$. The result follows now by the obvious consideration that $\mathrm{h}_{Z}(d)-\operatorname{dim}\left(\mathscr{G}_{1}^{\alpha}\right) \geq \mathrm{h}_{Z}(\alpha+1)-\operatorname{dim}\left(\mathscr{G}_{1}^{\alpha}\right)$ when $d>\alpha+1$

4.3. Almost linear pfaffian surfaces: the asymptotic region. We turn now our attention to the asymptotic case $\alpha \leq 10$. The proof of the following proposition relies on the degeneration lemma of [CF08] and on a computation done with the package Macaulay2, see GS. We postpone the latter part to the appendix.

Proposition 4.4. For $\alpha \leq 10$ and $d \geq \alpha+1$, a general surface of degree $d$ contains a linearly homogeneous subscheme $Z$ of order $\alpha$.

Thus, for $\alpha \leq 10$, a general surface of degree $d \geq \alpha+1$ is defined by an almost linear pfaffian of type $(\alpha, d-1-\alpha)$. In particular, a linearly homogeneous subscheme $Z$ of order $\alpha$ is asymptotic for $\alpha \leq 10$.

Proof. In view Remark 3.6, it suffices to check that a general surface $X_{d}$ of degree $d$ contains a linearly homogeneous subscheme of order $\alpha$.

Moreover, by CF08, Lemma 3.2], we need only check that a general surface $X_{d}$ of degree $d$ contains a linearly homogeneous subscheme of order $\alpha$ for $d$ in the range $\alpha+1, \ldots, 2 \alpha-2$. Recall also that, for $\alpha \leq 3$, the statement is well known, see CF08. Therefore, we must only prove that a general surface $X_{d}$ of degree $d$ contains a linearly homogeneous subscheme of order $\alpha$ for $4 \leq \alpha \leq 10$, and $d$ on the boundary line $d=2 \alpha-2$, and for the values summarized in the following table (including the boundary line $d=2 \alpha-2)$

\begin{tabular}{c|cccccccc}
$\alpha$ & $d$ & & & & & & & \\
\hline 4 & 5 & 6 & & & & & & \\
5 & 6 & 7 & 8 & & & & & \\
6 & 7 & 8 & 9 & 10 & & & & \\
7 & 8 & 9 & 10 & 11 & 12 & & & \\
8 & 9 & 10 & 11 & 12 & 13 & 14 & & \\
9 & 10 & 11 & 12 & 13 & 14 & 15 & 16 & \\
10 & 11 & 12 & 13 & 14 & 15 & 16 & 17 & 18
\end{tabular}

This is a finite number of verifications that can be performed by the package Macaulay2. We refer to the appendix for the outline of the algorithm. 
4.4. Almost linear pfaffian surfaces: the remaining non-asymptotic cases. We first work out the case $\alpha=15$, essentially due to Beauville. Its behavior is rather intriguing.

Proposition 4.5. Fix $\alpha=15$. Then for any $d$, a general surface of degree $d$ contains no linearly homogeneous subscheme of order $\alpha$, equivalently it is not defined by an almost homogeneous pfaffian of type $(15,1, d-16)$.

In particular, homogeneous subschemes of type $(15,1)$ are not asymptotic.

Proof. As in the proof of Proposition 4.3, we may consider only the case $d \geq \alpha+1=16$ and look at the function

$$
\psi(d)=\mathrm{h}_{Z}(d)-\operatorname{dim}\left(\mathscr{G}_{1}^{\alpha}\right) .
$$

Recall that $\operatorname{dim}\left(\mathscr{G}_{1}^{\alpha}\right)=4 \alpha^{2}-1$ so setting $\alpha=15$ we get $\operatorname{dim}\left(\mathscr{G}_{1}^{15}\right)=$ 899. On the other hand, in order to calculate $\mathrm{h}_{Z}(d)$, again we twist the resolution (3.1) by $\mathscr{O}_{\mathbb{P}^{3}}(d)$ and take global sections whereby computing $\mathrm{h}^{0}\left(\mathbb{P}^{3}, \mathscr{I}_{Z, \mathbb{P}^{3}}(d)\right)$. Using that, for $t \in \mathbb{N}$, one has $\left(\begin{array}{l}t \\ 3\end{array}\right)-\left(\begin{array}{c}t-1 \\ 3\end{array}\right)=\left(\begin{array}{c}t-1 \\ 2\end{array}\right)$, we get:

$$
\mathrm{h}^{0}\left(\mathbb{P}^{3}, \mathscr{I}_{Z, \mathbb{P}^{3}}(d)\right)=(2 \alpha+1)\left(\begin{array}{c}
d-\alpha+2 \\
2
\end{array}\right)+\left(\begin{array}{c}
d-2 \alpha+2 \\
3
\end{array}\right),
$$

and since $d \geq \alpha+1$ we have:

$$
\mathrm{h}^{0}\left(\mathbb{P}^{3}, \mathscr{I}_{Z, \mathbb{P}^{3}}(d)\right) \leq \frac{1}{2}(2 \alpha+1)(d-\alpha+2)(d-\alpha+1),
$$

so that:

$$
\mathrm{h}_{Z}(d) \geq\left(\begin{array}{c}
d+3 \\
3
\end{array}\right)-\frac{1}{2}(2 \alpha+1)(d-\alpha+2)(d-\alpha+1) .
$$

Setting $\alpha=15$ in the above formulas, we have:

$$
\psi(d) \geq \frac{1}{6}\left(d^{3}-87 d^{2}+2522 d-22314\right) .
$$

It is immediate to show that $\psi(d)$ is positive for all integers $d \geq 17$, so our statement follows for all $d \geq 17$. On the other hand, $\psi(16)$ is negative, so numerically one would expect the existence of a homogeneous subscheme of type $(15,1)$ on a general surface $S$ of degree 16 . But it is proved in Bea00, Proposition 7.6], that such a subscheme $Z$ cannot exists. This finishes the proof.

Remark 4.6. What is so surprising in the previous example? Let us go back to the notation introduced in Proposition 3.11. If $G$ is the component $\mathscr{G}_{1}^{15}$ of the Hilbert scheme which parametrizes linearly homogeneous subscheme of order 15 , and $G(16)$ is the corresponding incidence variety, $G(16)=\left\{\left(X_{16},[Z]\right) \mid Z \subset X_{16} \subset \mathbb{P}^{3}\right\}$, then $\psi(16)<0$ implies that $\operatorname{dim}(G(16))>\operatorname{dim}\left(\left|\mathscr{O}_{\mathbb{P}^{3}}(16)\right|\right)$ and one expects that the projection map $p_{16}: G(16) \rightarrow\left|\mathscr{O}_{\mathbb{P}^{3}}(16)\right|$ is dominant.

In fact, Beauville proves that the map does not dominate. A reason is that, in view of Theorem 2.1, the existence of $Z$ on a general surface $X$ of degree $d$ would imply the existence on $X$ of a rank 2 bundle $\mathscr{E}$, without intermediate cohomology (i.e. satisfying (1.1)), with an exact sequence:

$$
0 \rightarrow \mathscr{O}_{X} \rightarrow \mathscr{E} \rightarrow \mathscr{I}_{Z, X}(15) \rightarrow 0
$$


On the other hand, from the resolution it follows that $\mathrm{h}^{0}\left(X, \mathscr{I}_{Z, X}(15)\right)=31$, so that $\mathrm{h}^{0}(X, \mathscr{E})=32$ and a general global section of $\mathscr{E}$ determines on $X$ a deformation of $Z$. Thus the map $p_{16}$ of Proposition 3.11 has general fibers of dimension at least 31 , and this fact, numerically, implies that it cannot dominate. Indeed one computes that $\operatorname{dim}(G(16))=\operatorname{dim}(G)+\mathrm{h}^{0}\left(\mathbb{P}^{3}, \mathscr{I}_{Z, \mathbb{P}^{3}}(16)\right)-$ $1=899+92=991$, while $\operatorname{dim}\left(\left|\mathscr{O}_{\mathbb{P}^{3}}(16)\right|\right)=968$, so one expects that the image of $p_{16}$ has codimension 8 in $\left|\mathscr{O}_{\mathbb{P}^{3}}(16)\right|$. By applying Schreyer's algorithm described in the appendix of [Bea00], one finds that the codimension is indeed 8 (consequently, a general fiber of $p_{16}$ has dimension exactly equal to 31 ).

We have, thus, a counterexample to the naïve conjecture that the map $p_{d}$ of Proposition 3.11 is always of maximal rank, whatever the family $G$ is.

Problem. Although the previous example shows that one cannot expect that the map $p_{d}$ of Proposition 3.11 is always of maximal rank, there are possible restrictions on $G$ for which this maximal rank condition could be conjectured.

For instance, we know no examples of families $G$ of complete intersection zero-dimensional schemes in $\mathbb{P}^{3}$ for which the map $p_{d}$ fails to be of maximal rank. See also [CCG] for a discussion on this subject.

It remains to consider the situation for $11 \leq \alpha \leq 14$. For these values of $\alpha$, it turns out that there exist only a finite set of degrees $d$ such that a general surface of degree $d$ contains a homogeneous aG subscheme of type $(\alpha, 1)$. The following statements summarize the situation.

Proposition 4.7. Set $11 \leq \alpha \leq 14$. Then a general surface of degree $d$ contains a linearly homogeneous subscheme of order $\alpha$ if and only if $\alpha+1 \leq$ $d \leq 15$.

In particular, linearly homogeneous subschemes of order $\alpha$ are not asymptotic and a general surface of degree $d>15$ is not defined by an almost homogeneous pfaffian of type $(\alpha, 1, d-16)$, with $\alpha \geq 11$.

Proof. We first use the same argument of Proposition 4.5. Namely, in order to check that a general surface in $\mathbb{P}^{3}$ of degree $d$ does not contain a linearly homogeneous subscheme of order $\alpha$, for each $\alpha$ in the given range, we first note that $d$ must satisfy $d \geq \alpha+1$. Then we introduce the function $\psi(d)$ given in (4.3). Formulas 4.4 and (4.5) still hold here, and it is straightforward to deduce that, for each $\alpha$ in the given range, the function $\psi(d)$ is strictly positive for all integers $d \geq 16$. This settles the non-existence part.

On the other hand, we have to show that a general surface in $\mathbb{P}^{3}$ of degree $d$ does contain a linearly homogeneous subscheme of order $\alpha$, for $d=\alpha+1, \ldots, 15$. Namely, we have to check the statement for all values of $(\alpha, d)$ appearing in the table:

\begin{tabular}{c|cccc}
$\alpha$ & $d$ & & & \\
\hline 11 & 12 & 13 & 14 & 15 \\
12 & 13 & 14 & 15 & \\
13 & 12 & 15 & & \\
14 & 15 & & &
\end{tabular}


This is done with the aid of Macaulay2, using the same algorithm of Proposition 4.4, which we sketch in the appendix.

\section{Appendix. Almost linear pfaffian surfaces}

This appendix contains a description of the Macaulay2 routines that we used in order to prove that a general surface $X_{d} \subset \mathbb{P}^{3}$ of degree $d$ contains a homogeneous subscheme of type $(\alpha, 1)$ (i.e. a linearly homogeneous subscheme of order $\alpha)$ for the values of $(\alpha, d)$ appearing in Table 4.2 and in Table 4.6 .

It relies on a slight modification of Schreyer's algorithm appearing in the appendix to [Bea00], based on Adler's method, see [AR96]. For general reference on the package Macaulay2 one can consult the on-line documentation, see [GS].

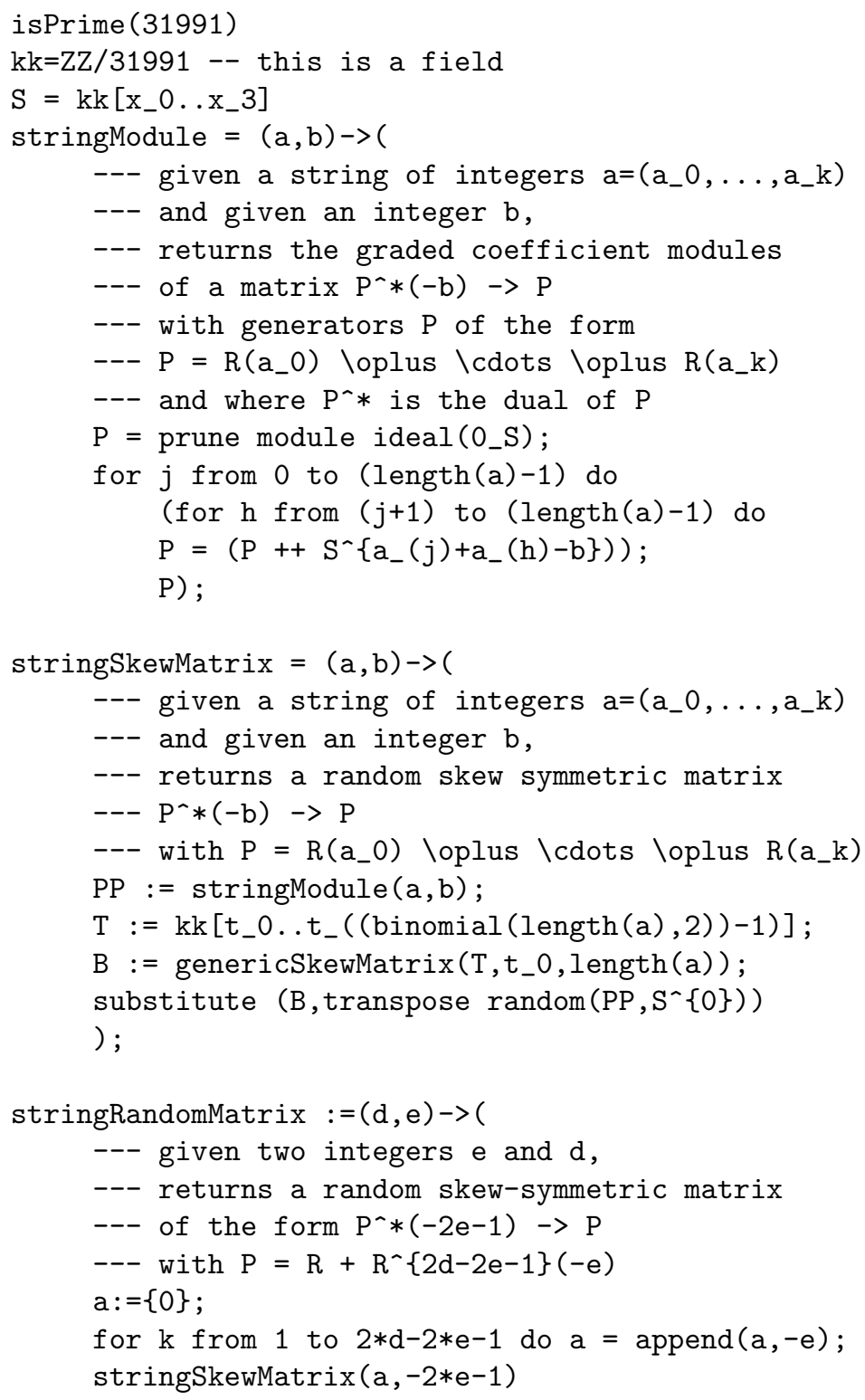




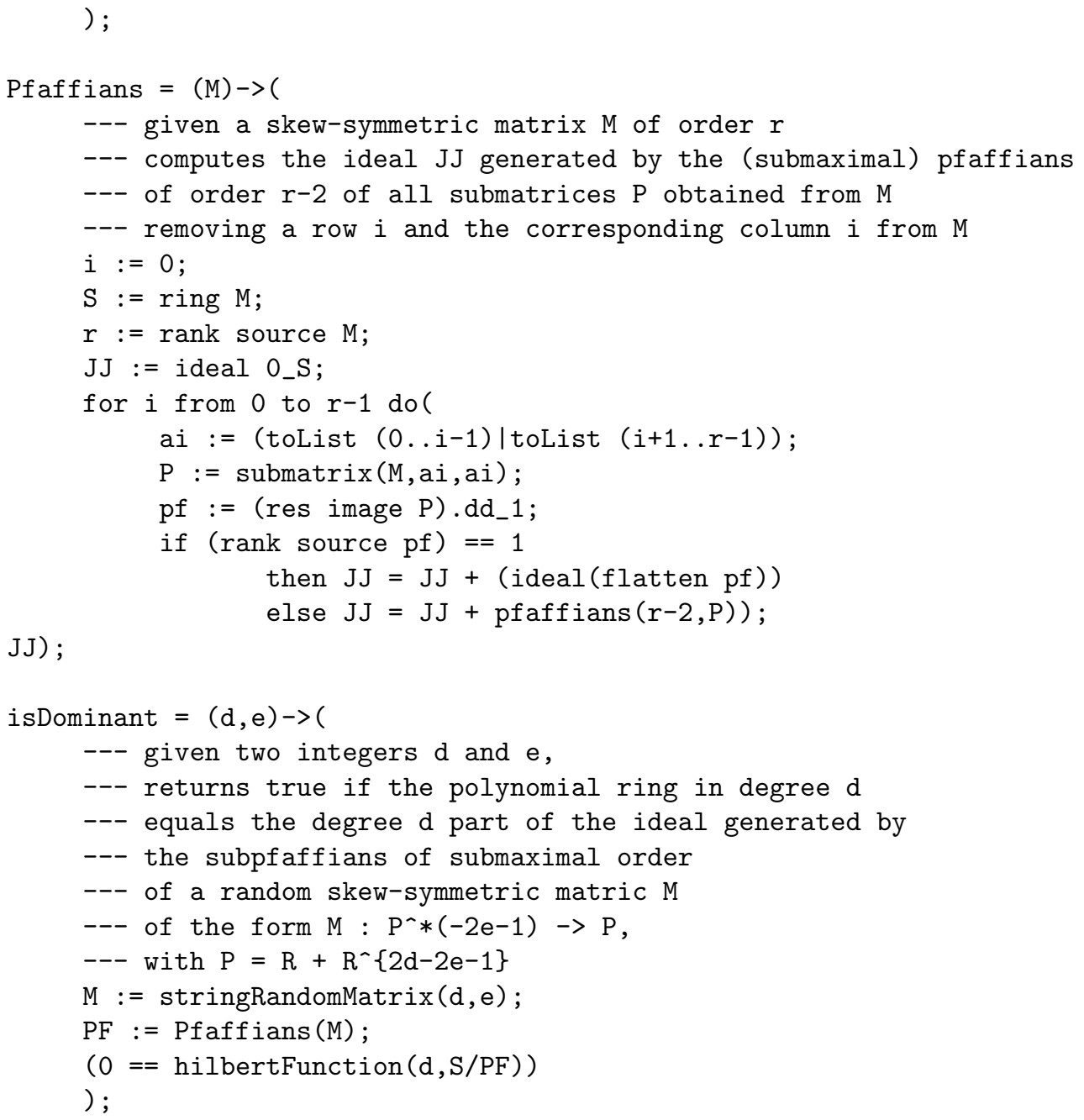

The variables $(d, e)$ used in the above algorithm are changed into the variables $(\alpha, d)$ used in Section 4 by the formula:

$$
e=d-\alpha-1 \text {. }
$$

One checks that the function isDominant above returns the value true for all the values of $(d, e)$ that correspond (under the equation $e=d-\alpha-1)$ to the values of $(\alpha, d)$ appearing in Table (4.2) and Table (4.6). This concludes the proof.

To perform the previous computations, on the server 3dom0 of Dipartimento di Matematica Ulisse Dini (Firenze), the time required was in any case shorter than 40 minutes.

\section{REFERENCES}

[AR96] Allan Adler and Sundararaman Ramanan, Moduli of abelian varieties, Lecture Notes in Mathematics, vol. 1644, Springer-Verlag, Berlin, 1996.

[Bea00] Arnaud Beauville, Determinantal hypersurfaces, Michigan Math. J. 48 (2000), 39-64, Dedicated to William Fulton on the occasion of his 60th birthday. 
[BE77] David A. Buchsbaum and David Eisenbud, Algebra structures for finite free resolutions, and some structure theorems for ideals of codimension 3, Amer. J. Math. 99 (1977), no. 3, 447-485.

[CF08] Luca Chiantini and Daniele Faenzi, Rank 2 acm bundles on a general quintic surface. Math. Nachr. to appear (2008).

[CCG] Enrico Carlini, Luca Chiantini and Anthony Geramita, On complete intersection points and general surfaces in $\mathbb{P}^{3}$. To appear on Mich. Math. J.(2008).

[CM00] LuCa Chiantini And Carlo Madonna, ACM bundles on a general quintic threefold, Matematiche (Catania) 55 (2000), no. 2, 239-258 (2002), Dedicated to Silvio Greco on the occasion of his 60th birthday (Catania, 2001). MR 1984199

[CM04] _ A splitting criterion for rank 2 bundles on a general sextic threefold, Internat. J. Math. 15 (2004), no. 4, 341-359.

[CM05] _ ACM bundles on general hypersurfaces in $\mathbb{P}^{5}$ of low degree, Collect. Math. 56 (2005), no. 1, 85-96.

[Die96] SusAn J. DiEsEL, Irreducibility and dimension theorems for families of height 3 Gorenstein algebras., Pac. J. Math. 172 (1996), no. 2, 365-397 (English).

[Fae08a] Daniele FAenzi, Rank 2 arithmetically Cohen-Macaulay bundles on a nonsingular cubic surface, J. Algebra 319 (2008), no. 1, 143-186.

[Fae08b] _ A Remark on Pfaffian Surfaces and aCM Bundles, to appear in Vector bundles and low codimensional subvarieties: state of the art and recent developments (2008) in the Series Quaderni di Matematica della Seconda Università di Napoli.

[GS] Daniel R. Grayson and Michael E. Stillman, Macaulay 2, a software system for research in algebraic geometry, Available at http://www.math.uiuc.edu/Macaulay2/

[IK99] Anthony IARRobino And Vassil Kanev, Power sums, Gorenstein algebras, and determinantal loci, Lecture Notes in Mathematics, vol. 1721, Springer-Verlag, Berlin, 1999, Appendix C by Iarrobino and Steven L. Kleiman.

[Kle78] Hans Kleppe, Deformation of schemes defined by vanishing of Pfaffians, J. Algebra 53 (1978), no. 1, 84-92.

[Kle98] JAN O. KLEPPE, The smoothness and the dimension of PGor $(H)$ and of other strata of the punctual Hilbert scheme, J. Algebra 200 (1998), no. 2, 606-628.

[Knö87] Horst KNÖRRER, Cohen-Macaulay modules on hypersurface singularities. I, Invent. Math. 88 (1987), no. 1, 153-164.

[MKRr07a] N. Mohan Kumar, Prabhakar A. RaO, and Girivau V. Ravindra, Arithmetically Cohen-Macaulay bundles on hypersurfaces, Comment. Math. Helv. 82 (2007), no. 4, 829-843.

[MKRR07b] _ Arithmetically Cohen-Macaulay bundles on three dimensional hypersurfaces, Int. Math. Res. Not. IMRN (2007), no. 8, Art. ID rnm025, 11.

[Ott88] Ottaviani, Giongio. Spinor bundles on quadrics. Trans. Amer. Math. Soc. 307 (1988), no. 1, 301-316.

E-mail address: chiantini@unisi.it

Dipartimento Di Scienze Matematiche e Informatiche R. Magari, Pian Dei Mantellini 44, I-53100 SienA, ItAly

$U R L:$ http://www2.dsmi.unisi.it/newsito/docente.php?id=4

E-mail address: daniele.faenzi@univ-pau.fr

Laboratoire de mathématiques et de leurs applications, Université de Pau et des Pays de L'Adour, Avenue de L'Université - BP 1155, 64013 Pau CEDEX FRANCE

$U R L:$ http://www.math.unifi.it/ faenzi 